\title{
Dynamique Non Linéaire Du Marché Boursier Marocain : Une Application Des Modèles A Changement De Régimes
}

\author{
N. Bensaid \\ Université Mohamed V, Faculté des sciences juridiques économiques et sociales, Agdal-Rabat
}

\begin{abstract}
In this paper, we study the dynamics of the Moroccan stock index the MASI as well as six Moroccan companies among the biggest market capitalizations. For this purpose, we characterize these dynamics by holding a particular class of the non-linear models, namely, the models with smooth transition «STAR» which are able to reproduce the switching regimes in the presence of transaction costs and in the presence of heterogeneous anticipations of the investors. The estimations done, conjugated to the theoretical framework, highlight the superiority of the process SETAR with regard to the linear model to reproduce the dynamics of the stock-exchange yields of the Moroccan companies, and to question the hypothesis of efficiency of financial markets.
\end{abstract}

Keywords: Non Linear Model-Switching Regime Model - Efficiency of financial markets

Résumé: Dans ce papier, nous étudions la dynamique de l'indice boursier marocain MASI ainsi que six sociétés marocaines parmi les plus grosses capitalisations boursières des secteurs bancaire et immobilier. A cette fin, nous caractérisons ces dynamiques en retenant une classe particulière des modèles non-linéaires en moyenne à savoir lesmodèles à transition lisse de type STAR qui sont en mesure de reproduire le comportement des régimesen présence de coûts de transaction hétérogènes et d'anticipations distinctes des opérateurs. Les résultats ainsi obtenus mettent en évidence la supériorité du processus ESTAR par rapport au modèle linéaire pour reproduire la dynamique des rentabilités boursières dessociétés marocaines, ils ont permis également de remettre en cause l'hypothèse d'efficience des marchés financiers en général, et l'efficience du marché financier marocain en particulier.

Mots- clés: Modèles non-linéaires- Changement de régimes- Efficience des marchés financiers

\section{Introduction}

Selon la célèbre définition de Fama quant à l'efficience des marchés financiers, il s'avère que les actifs financiers sont évalués conformément à leurs valeurs fondamentales. Or certains travaux rejettent cette conception en soulignant que les hypothèses sous-jacentes jugées restrictives de cette théorie (marchés parfaits, absences de coûts de transaction, gratuité de l'information, etc.) sontincompatibles avec l'existence de phénomènes de "mésalignement » durable des cours. D'autant plus qu'un certain nombre d'anomalies boursières sont liées aux différentes formes d'imperfections des marchés qui sont exclues à priori par l'hypothèse d'efficience. Ces frictions remettent parfaitement en cause la théorie de l'efficience des marchés financiers ainsi que l'hypothèse d'ajustement linéaire et symétrique des cours boursiers.

De ce fait, telle que définie parFama, l'hypothèse d'efficience se révèleincompatible avec le fonctionnement réel du marché en raison de l'hétérogénéité desanticipations des opérateurs sur le marché, de la présence des coûts de transaction etd'information distincts et de l'importance des imperfections du marché.

L'étude de l'évolution des cours boursiers par rapport aux fondamentaux est l'un des sujets les plus abordés dans la littérature. Elle a fait l'objet d'un nombre considérable de travaux qui se sont focalisés sur la modélisation de la dynamique d'ajustement des cours vers les fondamentaux et le test de l'hypothèse d'efficience. Ces travaux ont mobilisé des techniques économétriques distinctes, allant du plus simple au plus sophistiqué et du linéaire au non-linéaire.

\section{Supériorité Des Modèles Non Linéaires}

Plusieurs études empiriques ont montré que les variations des cours boursiers ne sont pas expliquées par les fondamentaux (Shiller (1981) et Jawadi (2004)). Jawadi (2006) montre que les cours semblent s'écarter durablement de leurs valeurs d'équilibre, ce qui induit une certaine persistance dans la dynamique des déviations des prix par rapport à l'équilibre.

Sur un plan empirique, il convient de noter que la modélisation des séries de rentabilités boursières par les modèles à changement de régime n'est pas une idée nouvelle, puisque l'on doit à Turner, Startz et Nelson (1989) et Van Norden et Schaller $(1993,1996)$ qui ont retenu la méthodologie d'Hamilton (1989) pour estimer les dynamiques boursières.

Mais, plus récemment Sarantis (2001) a mobilisé les modèles STAR pour explorer la dynamique des séries de rentabilités boursières annualisées des pays du G7, tandis que Jawadi (2001) a utilisé les processus à

DOI: 10.9790/5933-0705020915 $\quad$ www.iosrjournals.org $9 \mid$ Page


transition lisse pour étudier l'évolution des séries de rentabilités boursières du CAC40 et du S\&P500. Les résultats de ces études ont montré que les processus STAR sont plus appropriés que les modèles AR linéaires pour reproduire l'asymétrie et la persistance caractérisant les données boursières ainsi que les imperfections, les distorsions et les effets d'inertie induits par les coûts de transactions. Au total, les auteurs ont rejeté l'hypothèse de linéarité en faveur d'une alternative non-linéaire de type STAR et ont montré la supériorité de ces processus par rapport aux modèles linéaires pour expliquer l'inefficience du marché et notamment pour spécifier la dynamique alternative du processus non-linéaire généré par les données boursières étudiées.

\section{Justification Et Choix Des Modèles STAR :}

L'idée des modèles STAR étant de reproduire la transition entre les régimes qui sont délimités par les seuils spécifiques ainsi générés par l'hétérogénéité des coûts de transaction et le reflet de la divergence des anticipations et des réactions des intervenants sur le marché.Les modèles STAR paraissent appropriés notamment pour reproduire l'ajustement des prix en présence de coûts de transaction hétérogènes.Ces modèles sont les mieux adaptés à l'étude des séries boursières pour trois raisons :

1- L'étude de la dynamique des indices boursiers concerne des actions distinctes à cô̂ts d'arbitrage différents : L'hypothèse d'efficience informationnelle exclut tout décalage temporel entre la divulgation de l'information et son intégration dans les prix dans la mesure où les coûts d'information et de transaction sont nuls. L'ajustement des cours est alors instantané. Néanmoins la réaction des opérateurs du marché et l'ajustement des prix ne sont pas forcément instantanés. La présence des coûts de transaction est une des raisons qui expliquent ce décalage. Il apparaît que ces coûts présentent une limite à l'arbitrage et à l'efficience dans la mesure où ils induisent des effets d'inertie et une forme de persistance dans la dynamique des cours, ces coûts peuvent freiner les transactions boursières susceptibles de ramener le cours à sa valeur fondamentale. Les déviations des cours peuvent suivre un processus non-linéaire avec retour à la moyenne, dont la vitesse de convergence augmente avec l'ampleur du déséquilibre.

2- Hétérogénéité des anticipations :Il existe différents intervenants sur le marché dont les degrés de perception et de compréhension sont différents. On peut citer les fondamentalistes, les chartistes, les bruiteurs etc... Ainsi, il existe des opérateurs plus informés que d'autres, ce qui explique des horizons d'investissement différents. Selon les fondamentalistes chaque titre dispose d'une valeur fondamentale autour de laquelle fluctuent les prix. Ces derniers ramènent les cours vers leur valeur fondamentale, ce qui explique des périodes de haute volatilité observée sur le marché. Les chartistes, de leur côté, agissent sur le marché en se basant sur les prix passés ; leur action contribue à accroître l'écart entre les prix et les fondamentaux. De ce fait, les interactions entre chartistes et fondamentalistes peuvent générer un prix qui est loin d'être le reflet de la réalité économique. Ces interactions peuvent impliquer des périodes de hausses éphémères et des périodes de récessions profondes et violentes. $\mathrm{Ce}$ qui peut induire des délais d'ajustement des cours lorsque ceux-ci tentent de rejoindre leurs valeurs d'équilibre.

3- Mimétisme selon l'approche keynésienne : la valeur d'un titre sur un marché financier est interprétée comme la préférence moyenne de l'ensemble des opérateurs. On peut en déduire que la rationalité d'un agent devrait le conduire à se procurer le titre qui est préféré par la majorité. Dans ce cas de figure, la manipulation du marché par des opérateurs devient possible, puisqu'en achetant massivement un titre donné, ces opérateurs provoquent une hausse de son cours. Le processus de hausse des prix dure tant que le nombre d'acquéreurs croît : c'est l'effet boule de neige qui reflète un comportement mimétique. Le marché boursier devient alors un lieu où les croyances collectives et les psychologies sociales jouent un rôle très important dans la formation des cours. Il est l'équivalent d' "une entité collective qui émerge sur la base d'un réseau serré d'anticipations croisées et d'influences interpersonnelles ». En présence du mimétisme, le cours ne reflète plus les fondamentaux mais plutôt les anticipations des investisseurs concernant l'opinion moyenne des marchés. Dans un tel contexte, les opérateurs sont incités à suivre l'opinion moyenne du marché, et à ne pas s'en écarter, car le fait de s'en écarter engendre un risque. Les opérateurs vont ainsi s'intéresser à l'anticipation de la psychologie de masse et de l'opinion du marché, et non aux fondamentaux des titres; Cela peut induire une bulle rationnelle mimétique. Cette bulle mimétique est vulnérable dans la mesure où tout changement de l'opinion moyenne du marché peut engendrer son éclatement. Les techniques de modélisation linéaire ne prennent pas en considération de tels comportements.

\section{Présentation Des Modèles STAR :}

Les modèles à seuil (TAR : Thresholdautoregressive) permettent de capter les changements de régime observés dans l'évolution des séries financières et de les modéliser. Lorsque la variable de seuil est la même que celle à expliquer, le modèle s'appelle SETAR (Self-ExcitingThresholdAutoRegressive).

Les processus TAR permettent donc, dans une certaine mesure, de raconter une histoire économique. Ils n'en 
présentent pas moins certains défauts notamment sur deux points :

-La discontinuité du processus au niveau du seuil complique l'estimation et les tests.

-Ils peuvent générer des révisions de prévisions peu crédibles. Une faible variation des conditions initiales est susceptible d'entraîner la substitution d'une équation par une autre notamment lorsque $\mathbf{y}$ est proche du seuil $\mathbf{c}$. La solution est d'autoriser une transition douce entre les régimes.

Les processus STAR ont été introduits par Teräsvirta et Anderson (1992) et Teräsvirta (1994). Ils constituent une généralisation des modèles à seuil à transition brutale (modèles TAR) de Chan et Tong (1986). Les modèles STAR définissent deux régimes extérieurs entre lesquels la transition est supposée être lisse, et un continuum d'états intermédiaires entre ces régimes. Les modèles à transition lisse sont plus appropriés que les modèles linéaires standards parce qu'ils ne restreindrent pas les dynamiques de ces régimes à être symétriques. En outre, ils peuvent reproduire les mouvements asymétriques et irréguliers caractérisant les données boursières.

Un modèle STAR peut être défini comme suit :

$$
\mathbf{Y}_{t}=\left(\alpha_{0}+\alpha_{1} \mathbf{Y}_{t-1}+\cdots+\alpha_{p} \mathbf{Y}_{t-p}\right)+\left(\beta_{0}+\beta_{1} \mathbf{Y}_{t-1}+\cdots+\beta_{p} \mathbf{Y}_{t-p}\right) X \mathbf{F}\left(\mathbf{s}_{t}, \gamma, \mathbf{c}\right)+\varepsilon_{t}
$$

Où $\varepsilon t \rightarrow \mathrm{N}(0, \sigma 2)$, $\gamma$ désigne la vitesse de transition $\gamma>\mathbf{0}$, st est la variable de transition et $\mathbf{c}$ est le paramètre de seuil.

Le modèle utilisé dans notre travail est un modèle non linéaire de type SETAR car la variable à expliquer est elle-même la variable du seuil, l'équation du modèle s'écrit comme suit :

$$
\begin{cases}Y=c_{1}+b_{1} x & \text { si } Y>T \\ Y=c_{2}+b_{2} x & \text { si } Y<T\end{cases}
$$

Où

Y : la variable à expliquer (le rendement dans notre cas), considérée aussi comme la variable du seuil (elle détermine le régime).

$\mathrm{X}$ : la variable explicative (l'indice boursier MASI dans notre cas)

$\mathrm{C}_{1}, \mathrm{c}_{2}, \mathrm{~b}_{1}, \mathrm{~b}_{2}$ sont les paramètres du modèle à estimer.

\section{L'application Du Modèle SETAR Au Marché Boursier Marocai}

Nous nous intéressons à présent à la modélisation des processus SETAR pour appréhender l'asymétrie et la non-linéarité inhérentes aux dynamiques des séries boursières relatives aux valeurs cotées sur le marché boursier marocain. Sera étudiée la dynamique de l'indice boursier marocain à savoir le MASI ainsi que celle des six sociétés cotées parmi les grosses capitalisations des secteurs de l'immobilier et du bancaire. Nous examinons la capacité de ces processus à révéler les irrégularités et les ruptures présentes dans le comportement des cours boursiers. L'idée sous-jacente consiste à vérifier l'existence d'une relation non-linéaire sur une période déterminée, mais linéaire par sous-périodes.

\section{Description Des Donnees :}

La base de données comprend le rendement jour par jour des six sociétés cotées parmi les grosses capitalisations boursières ainsi que l'indice du marché MASI pour la période globale allant du 07/07/2006 au 02/09/2015. Les rendements sont obtenus comme première différence de la série logarithmique des cours.

\section{Statistiques Descriptives :}

\begin{tabular}{|l|l|l|l|l|l|l|l|}
\hline Série & Moyenne & Ecart-Type & Max & Min & Skewnes & Kurtosis & Jaque-Bera \\
\hline MASI & 10823,67 & & 14925,99 & 7039,32 & 0,40 & $-0,21$ & \\
\hline BCP & 0,07 & & 15,82 & $-6,19$ & 0,57 & 9,22 & 0,0001 \\
\hline ATW & 0,01 & 1,27 & 5,83 & $-6,18$ & 3,59 & 0,07 & 0,0064 \\
\hline BMCE & 0,00 & 1,56 & 5,83 & $-6,19$ & 3,52 & 0,03 & 0,002 \\
\hline ADDOHA & $-0,10$ & 1,73 & 8,51 & $-10,54$ & 4,04 & $-0,25$ & 0,0056 \\
\hline ADI & $-0,18$ & 2,07 & 9,53 & $-10,54$ & 9,36 & $-0,95$ & 0,00167 \\
\hline CGI & $-0,10$ & 2,04 & 9,21 & $-10,54$ & 3,64 & $-0,39$ & 0,003 \\
\hline
\end{tabular}

Sur la période d'étude, le rendement moyen des entreprises bancaires paraît positif, il est caractérisé par une certaine stabilité contrairement à celui des entreprises immobilières qui enregistre plutôt une tendance baissière.

L'écart type mesure le degré de volatilité du rendement des entreprises étudiées. Le secteur bancaire enregistre moins de volatilité que le secteur immobilier. 
L'analyse du Skewness et du Kurtosis complétée par JacqueBera nous conduit à des conclusions habituelles dans les études des cours boursiers. Elles sont différentes de 0 et 3 , cequi signifie que la distribution n'est pas normale mais plutôt asymétrique.

\section{Stationnarité des séries :}

Toutes les séries sont stationnaires à l'exception d'ADI et du MASI qui sont stationnaires en première différence, le tableau ci-après résume le degré de stationnarité de chaque série.

\begin{tabular}{|l|l|l|}
\hline Série & Stationnarité & degré de stationnarité \\
\hline MASI & Oui & $\mathbf{1}$ \\
\hline ADH & Oui & $\mathbf{0}$ \\
\hline ADI & Oui & $\mathbf{1}$ \\
\hline CGI & Oui & $\mathbf{0}$ \\
\hline AWB & Oui & $\mathbf{0}$ \\
\hline BMCE & Oui & $\mathbf{0}$ \\
\hline BCP & Oui & $\mathbf{0}$ \\
\hline
\end{tabular}

Les rendements à l'instant t pour les entreprises bancaires sont expliqués par les rendements antérieurs jusqu'à j-6, alors que ceux des entreprises immobilières sont expliqués par ceux antérieurs jusqu'à j-3 en moyenne. Ceci dit que les cours des entreprises bancaires suivent quasi parfaitement la tendance du marché contrairement aux cours des entreprises immobilières qui sont expliqués par d'autres facteurs (fondamentaux ou autres).

\section{L'équation du modèle :}

Les résultats de la modélisation ont permis de relever deux régimes au moins pour l'ensemble des séries de notre échantillon, ceci témoigne de la non linéarité des séries de rentabilité et l'ajustementasymétrique de $Y_{t}$ par rapport à ses valeurs passées en fonction de la variable detransition, $Y_{t-1}$ qui permet de détecter les signes de déviation ainsi que leur ampleur, les résultats de la modélisation sur les différents régimes de chaque entreprise sont résumés dans le tableau ci-après:

\begin{tabular}{|c|c|c|c|}
\hline Secteurs & Entreprises & Equations du modèle & Interprétations \\
\hline \multirow{3}{*}{ 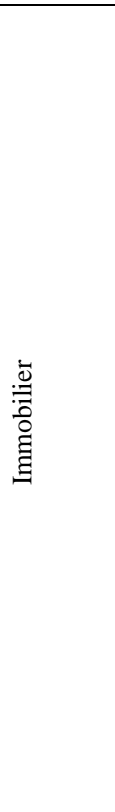 } & ADDOHA & 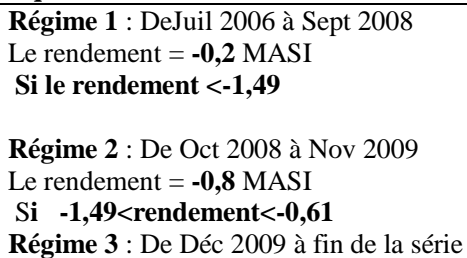 & $\begin{array}{l}\text { La modélisation a permis de relever } 3 \text { régimes. } \\
\text { Le rendement est expliqué parfaitement par } \\
\text { l'évolution du MASI lorsque le rendement ne } \\
\text { dépasse pas les deux premiers régimes. Dans } \\
\text { les autres casle rendement est expliqué par les } \\
\text { fondamentaux de l'entreprise ou autres. } \\
\text { Ilest à noter que la vitesse moyenne de } \\
\text { transitionest de } \mathbf{0 , 7 5} \text {. }\end{array}$ \\
\hline & ADI & $\begin{array}{l}\text { Régime } \mathbf{1} \text { : De Juil } 2006 \text { à Sept } 2009 \\
\text { Le rendement = -0,3 MASI } \\
\text { Si le rendement }<\mathbf{- 1 , 2 5} \\
\text { Régime } \mathbf{2} \text { : De Oct } 2009 \text { à Fin de la série }\end{array}$ & $\begin{array}{l}\text { La modélisation a permis de relever } 5 \text { régimes. } \\
\text { Le rendement est expliqué parfaitement par } \\
\text { l'évolution du MASI lorsque le rendement ne } \\
\text { dépasse pas le premier régime. Autrement le } \\
\text { rendement est expliqué par d'autres facteurs. } \\
\text { Il est à préciser que la vitesse moyenne de } \\
\text { transition est de } \mathbf{0 , 6 4} \text {. }\end{array}$ \\
\hline & CGI & $\begin{array}{l}\text { Régime } \mathbf{1} \text { : De Juil } 2006 \text { à Nov } 2008 \\
\text { Le rendement=-0,3 MASI } \\
\text { Si le rendement }<-\mathbf{1 , 6 6} \\
\text { Régime } \mathbf{2} \text { : De Déc } 2008 \text { à Nov } 2009 \\
\text { Le rendement =-0,7 MASI } \\
\text { Si } \mathbf{- 1 , 6 < \text { rendement<-0,15 }} \\
\text { Régime } \mathbf{3} \text { : De Déc } 2009 \text { à fin de la série }\end{array}$ & $\begin{array}{l}\text { La modélisation a permis de relever } 5 \text { régimes. } \\
\text { Le rendement est expliqué parfaitement par } \\
\text { l'évolution du MASI lorsque le rendement ne } \\
\text { dépasse pas les deux premiers régimes. Ailleurs } \\
\text { le rendement est expliqué pard'autres } \\
\text { déterminants. } \\
\text { La vitesse moyenne de transition est de } \mathbf{0 , 6 1} \text {. }\end{array}$ \\
\hline 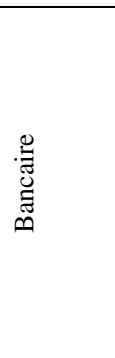 & AWB & 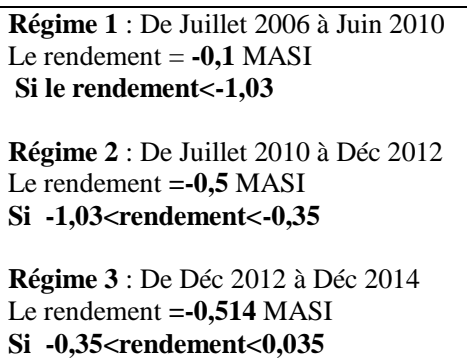 & $\begin{array}{l}\text { La modélisation a permis de relever } 5 \text { régimes. } \\
\text { Le rendement est expliqué parfaitement par } \\
\text { l'évolution du MASI dans } 4 \text { régimes parmi } 6 \text {. } \\
\text { La valeur d'ATW suit parfaitement la tendance } \\
\text { du marché, son évolution est significativement } \\
\text { liée à l'évolution du MASI. } \\
\text { La vitesse moyenne de transition est de } \mathbf{0 . 8 6} \text {. }\end{array}$ \\
\hline
\end{tabular}




\begin{tabular}{|c|c|c|}
\hline & $\begin{array}{l}\text { Régime } 4 \text { : De Déc } 2014 \text { Juillet } 2015 \\
\text { Le rendement }=\mathbf{0 , 7 4 5 1 4} \text { MASI } \\
\text { Si } \mathbf{0 , 0 3 5}<\text { rendement }<\mathbf{1 , 0 7 5} \\
\text { Régime } 5 \text { et } \mathbf{6} \text { : Non significatif pour le } \\
\text { reste de la période }\end{array}$ & \\
\hline BMCE & 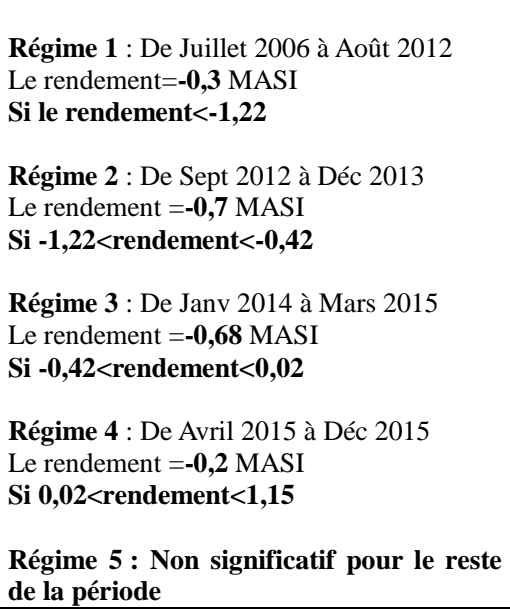 & $\begin{array}{l}\text { La modélisation a permis de relever } 5 \text { régimes. } \\
\text { Le rendement est expliqué parfaitement par } \\
\text { l'évolution du MASI pour } 4 \text { régimes parmi } 6 \text {. La } \\
\text { valeur de BMCE est significativement liée à la } \\
\text { bourse. } \\
\text { La vitesse moyenne de transition est de } \mathbf{0 . 8 5} \text {. }\end{array}$ \\
\hline $\mathrm{BCP}$ & $\begin{array}{l}\text { Régime } 1 \text { : De Juillet } 2006 \text { à Janv } 2009 \\
\text { Le rendement=-0,7MASI } \\
\text { Si le rendement }<-\mathbf{0 , 8 1} \\
\text { Régime } \mathbf{2} \text { : De Fev } 2009 \text { à Janv2014 } \\
\text { Le rendement=-1,23MASI } \\
\text { Si -0,81<le rendement<0,02 } \\
\text { Régimes } 3 \text { et } \mathbf{4} \text { :Non significtatif }\end{array}$ & $\begin{array}{l}\text { La modélisation a permis de relever } 4 \text { régimes. } \\
\text { Le rendement expliqué parfaitement par } \\
\text { l'évolution du MASI lorsqu'il ne dépasse pas les } \\
\text { deux premiers régimes. Ailleurs, d'autres } \\
\text { facteurs peuvent expliquer le rendement. } \\
\text { La vitesse moyenne de transition est de } \mathbf{0 , 7 9 4}\end{array}$ \\
\hline
\end{tabular}

\section{La validation du modèle :}

Le modèle est significatif vu que les statistiques de Fisher sont toutes inférieures à $5 \%$. Pour compléter la validation du modèle, nous avons effectué le test d'autocorrélation des résidus, le tableau ci-après récapitule les résultats de ce test :

\begin{tabular}{|l|l|l|l|l|l|l|}
\hline \multicolumn{7}{|c|}{ TEST DE d'autocorrélation LM (autocorrélation des résidus) } \\
\hline & ATW & BCP & BMCE & ADH & ADI & CGI \\
\hline P-value Fisher & $\mathbf{0 , 7 8}$ & $\mathbf{0 , 6 1}$ & $\mathbf{0 , 2 5}$ & $\mathbf{0 , 0 0 0 2}$ & $\mathbf{0}$ & $\mathbf{0 , 0 2 3}$ \\
& & & & & & \\
& & & & & & \\
\hline
\end{tabular}

Source : Elaboré par nos propres soins à partir des sorties du logiciel Eviews

Pour le secteur bancaire, l'autocorrélation des résidus est non significative au seuil de $5 \%$ ce qui justifie que le rendement des entreprises bancaires est expliqué parfaitement par l'indice boursier MASI. L'autocorrélation des résidus pour le secteur immobilier est fortement significative, ceci montre que d'autres variables contribuent à l'explication du rendement des entreprises immobilières autres que le MASI.

\section{Conclusion}

Le présent travail avait pour objectif d'étudier la dynamique des cours boursiers des plus grosses capitalisations boursières marocaines, en ayant recours à la méthodologie des modèles à changement de régime. Ces processus permettent à l'ajustement d'être asymétrique et variable dans le temps. Ils définissent des dynamiques distinctes et des régimes différents selon que le cours est loin ou non de l'équilibre.

Le recours à cette méthodologie est justifié principalement par quelques imperfections du marché à savoir : La présence des coûts de transaction ainsi que l'hétérogénéité comportementale qui caractérisent les marchés boursiers, remettant ainsi en cause la définition standard de l'hypothèse d'efficience des marchés donnée par «Fama, et qui sont à l'origine des mésalignements durables des cours.

Les estimations effectuées ont montré que les modèles à changement de régime étaient appropriés pour reproduire la dynamique des cours de l'indice MASI et des plus grosses capitalisations du marché marocain. Les résultats ont ainsi confirmé l'existence de deux régimes au moins pour l'ensemble des séries. 
Il est à préciser que les entreprises du secteur bancaire se caractérisent par plus de stabilité et leurs rendements sont expliqués parfaitement par l'évolution du MASI, contrairement aux entreprises du secteur immobilier qui paraissent plutôt volatiles et leurs rendements sont expliqués en sus du MASI par d'autres facteurs (fondamentaux de l'entreprise ou autres).

L'existence de plusieurs régimes nous permet de constater un ajustement asymétrique des cours par rapport à leurs valeurs fondamentales. Elle nous permet aussi de remettre en question l'efficience du marché boursier marocain ou plutôt de constater son inefficience.

\section{Annexes}

Tabl 1 : Périodes d'études et Nombre d'observations

\begin{tabular}{|l|l|l|}
\hline Séries & Période de l'étude & Nombre d'observations \\
\hline ATW & $\mathbf{0 7 / 0 7 / 2 0 0 6 - 0 2 / 0 9 / 2 0 1 5}$ & $\mathbf{1 7 7 2}$ \\
\hline BCP & $\mathbf{0 7 / 0 7 / 2 0 0 6 - 0 2 / 0 9 / 2 0 1 5}$ & $\mathbf{1 7 7 2}$ \\
\hline BMCE & $\mathbf{0 7 / 0 7 / 2 0 0 6 - 0 2 / 0 9 / 2 0 1 5}$ & $\mathbf{1 7 7 2}$ \\
\hline ADDOHA & $\mathbf{0 7 / 0 7 / 2 0 0 6 - 0 2 / 0 9 / 2 0 1 5}$ & $\mathbf{1 7 7 2}$ \\
\hline ADI & $\mathbf{0 7 / 0 7 / 2 0 0 6 - 0 2 / 0 9 / 2 0 1 5}$ & $\mathbf{1 7 7 2}$ \\
\hline CGI & $\mathbf{0 7 / 0 7 / 2 0 0 6 - 0 2 / 0 9 / 2 0 1 5}$ & $\mathbf{1 7 7 2}$ \\
\hline MASI & $\mathbf{0 7 / 0 7 / 2 0 0 6 - 0 2 / 0 9 / 2 0 1 5}$ & $\mathbf{1 7 7 2}$ \\
\hline
\end{tabular}

Tabl 2 : Résultats de spécification des modèles AR

\begin{tabular}{|l|l|l|l|l|l|l|}
\hline Valeur & ATW & BCP & BMCE & ADDOHA & ADI & CGI \\
\hline Retard optimal $(\mathrm{p})$ & $\mathbf{6}$ & $\mathbf{4}$ & $\mathbf{5}$ & $\mathbf{4}$ & $\mathbf{2}$ & $\mathbf{2}$ \\
\hline
\end{tabular}

Evolution des cours entre 2006 et 2015

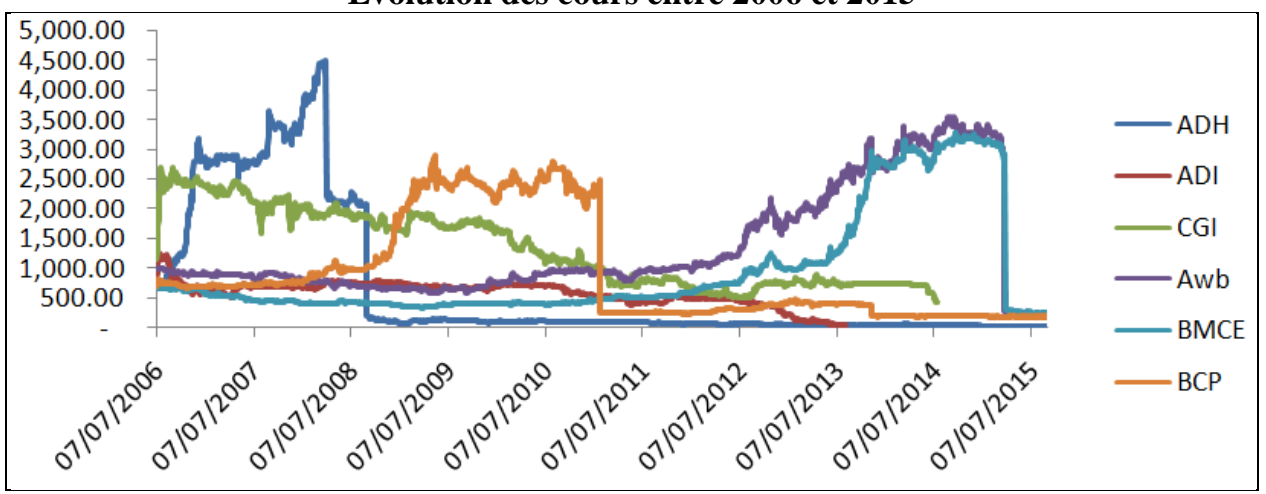

Comparaison entre la série observée et la série estimée :

\section{- ADDOHA}

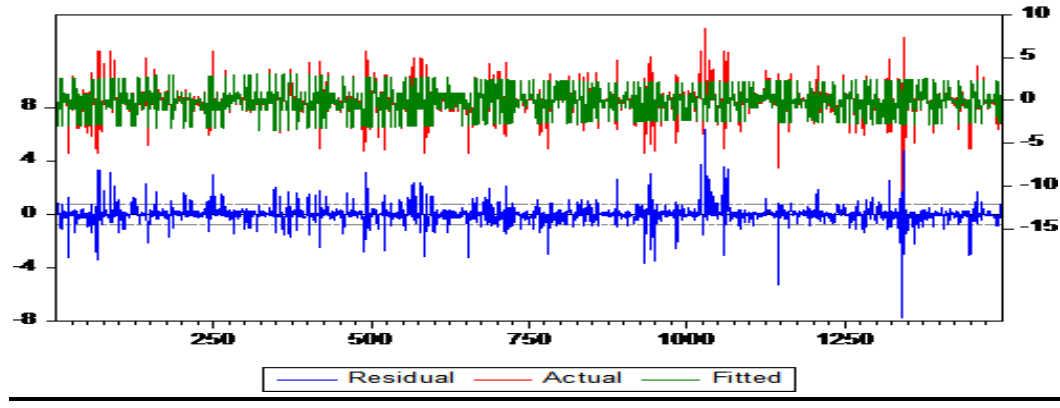

- Alliance Développement Immobilier:

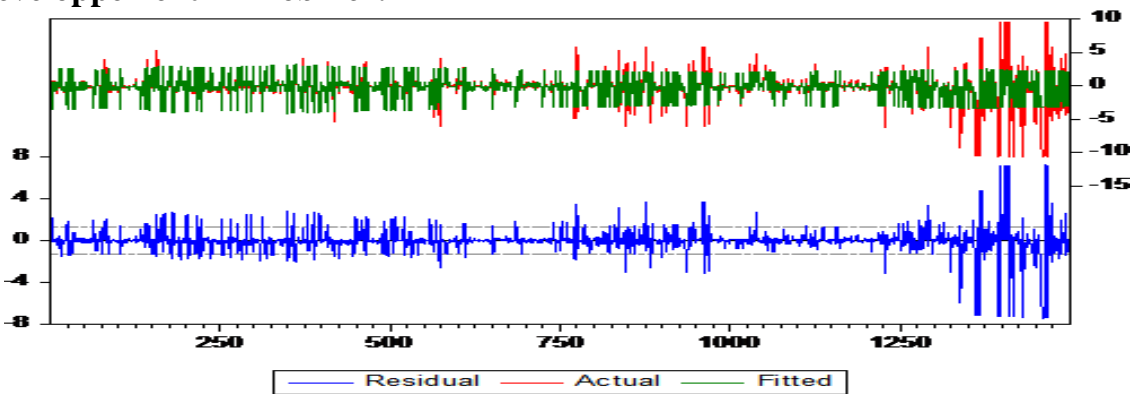


- Compagnie Générale Immobilière:

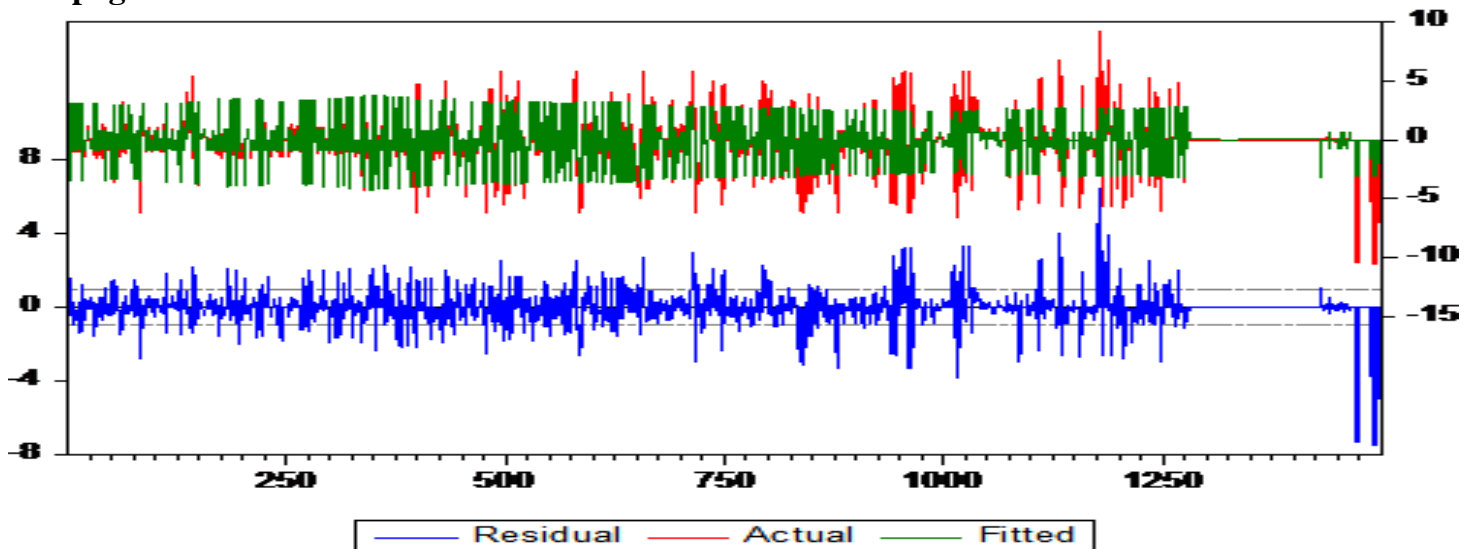

- ATTIJARI WAFABANK

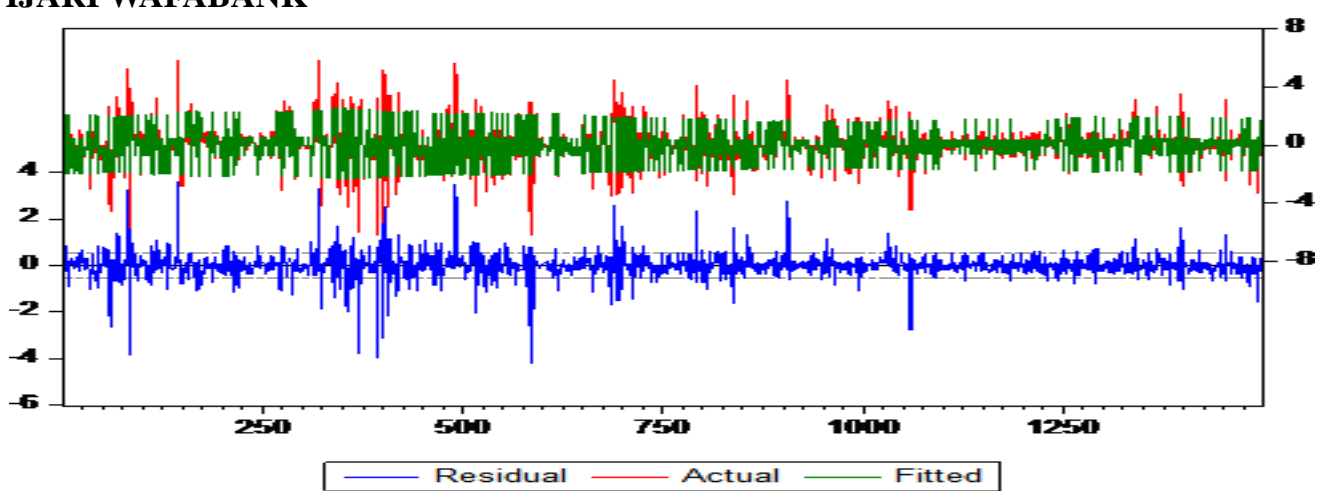

- BMCE

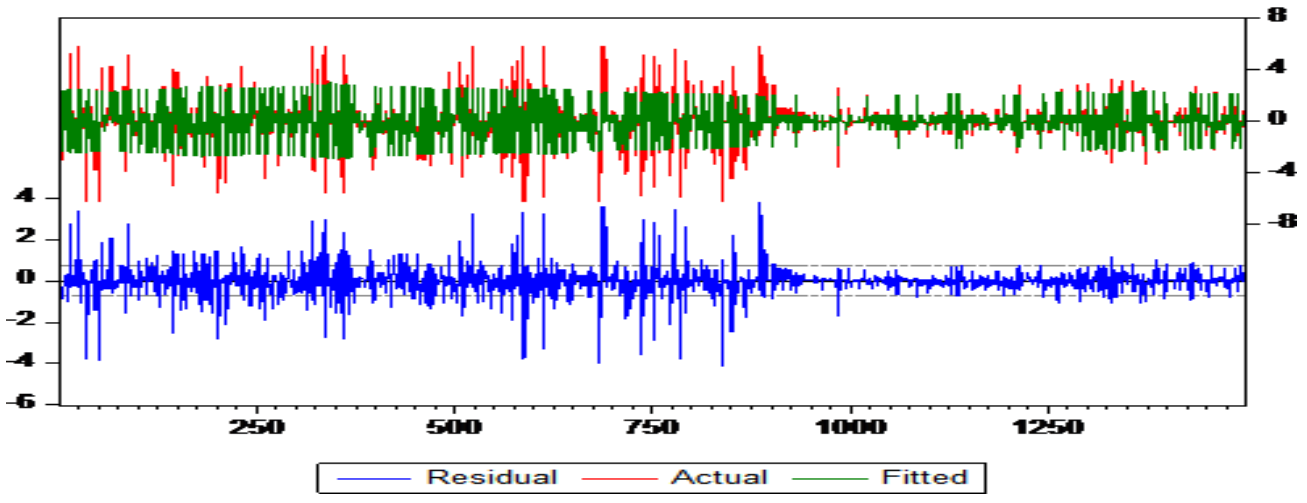

- $\quad \mathbf{B C P}$

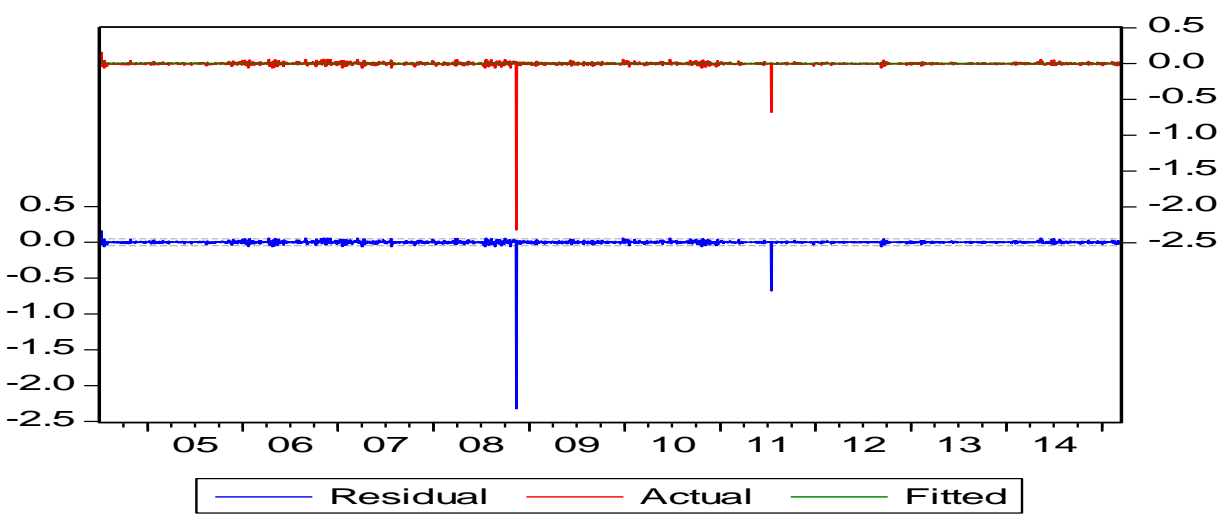

